\title{
Increased production of L-serine in Escherichia coli through Adaptive Laboratory Evolution
}

Mundhada, Hemanshu; Seoane, Jose Miguel; Schneider, Konstantin; Christensen, Hanne Bjerre; Koza, Anna; Herrgard, Markus; Feist, Adam; Nielsen, Alex Toftgaard

Published in:

Metabolic Engineering

Link to article, DOI:

10.1016/j.ymben.2016.11.008

Publication date:

2017

Document Version

Peer reviewed version

Link back to DTU Orbit

Citation $(A P A)$ :

Mundhada, H., Seoane, J. M., Schneider, K., Christensen, H. B., Koza, A., Herrgard, M., Feist, A., \& Nielsen, A. T. (2017). Increased production of L-serine in Escherichia coli through Adaptive Laboratory Evolution. Metabolic Engineering, 39, 141-150. https://doi.org/10.1016/j.ymben.2016.11.008

\section{General rights}

Copyright and moral rights for the publications made accessible in the public portal are retained by the authors and/or other copyright owners and it is a condition of accessing publications that users recognise and abide by the legal requirements associated with these rights.

- Users may download and print one copy of any publication from the public portal for the purpose of private study or research.

- You may not further distribute the material or use it for any profit-making activity or commercial gain

- You may freely distribute the URL identifying the publication in the public portal 


\title{
Increased production of L-serine in Escherichia coli through Adaptive
}

\section{Laboratory Evolution}

Hemanshu Mundhada ${ }^{1}$, Jose S. Miguel ${ }^{1}$, Konstantin Schneider ${ }^{1}$, Anna Koza ${ }^{1}$, Hanne B. Christensen ${ }^{1}$, Tobias Klein $^{1}$, Patrick V. Phaneuf ${ }^{2}$, Markus Herrgard ${ }^{1}$, Adam M. Feist ${ }^{1,2}$ and Alex T. Nielsen ${ }^{* 1}$

${ }^{1}$ Novo Nordisk Foundation Center for Biosustainability, Technical University of Denmark, $2800 \mathrm{Kgs}$. Lyngby, Denmark

${ }^{2}$ Bioengineering, University of California, San Diego, La Jolla, CA, USA

*Corresponding Author, Prof. Alex Toftgaard Nielsen, The Novo Nordisk Foundation Center for Biosustainability, Technical University of Denmark, Kemitorvet, Building 220, Room 308F, 2800 Kgs. Lyngby, Denmark. Phone +45 45258010. atn@biosustain.dtu.dk

\begin{abstract}
L-serine is a promising building block biochemical with a high theoretical production yield from glucose. Toxicity of L-serine is however prohibitive for high-titer production in E. coli. Here, E. coli lacking L-serine degradation pathways was evolved for improved tolerance by gradually increasing L-serine concentration from 3 to $100 \mathrm{~g} / \mathrm{L}$ using adaptive laboratory evolution (ALE). Genome sequencing of isolated clones revealed multiplication of genetic regions, as well as mutations in thrA, thereby showing a potential mechanism of serine inhibition. Other mutations were evaluated by MAGE combined with amplicon sequencing, revealing role of $r h o, l r p, p y k F$, eno, and $r p o B$ on tolerance and fitness in minimal medium. Production using the tolerant strains resulted in $37 \mathrm{~g} / \mathrm{L}$ of $\mathrm{L}-$ serine with a $24 \%$ mass yield. The resulting titer is similar to the highest production reported for any organism thereby highlighting the potential of ALE for industrial biotechnology.
\end{abstract}

\section{Abbreviations}

Adaptive Laboratory Evolution (ALE); Mutiplex Genome Engineering (MAGE).

Keywords: Adaptive laboratory evolution (ALE); Serine tolerance; Serine production; Fermentation; E. coli

\section{Introduction}

L-serine has been proposed as a potential building block biochemical for production of a range of chemicals and materials (Werpy and Petersen, 2004). Although L-serine has the highest theoretical production yield 
from glucose of all amino acids, the actual yield that has been achieved through fermentation ranks among the lowest (Becker and Wittmann, 2012). L-serine is currently used in cosmetic and pharmaceuticals industries with an estimated annual demand of 400 tons (Leuchtenberger et al., 2005). An efficient serine production strain could also be used as a platform for the production of ethanolamine and renewable ethylene glycol (Pereira et al., 2016). It is therefore highly attractive to produce L-serine at high yields and titers from glucose. Recently, we demonstrated high yield serine production using an engineered E. coli strain. Two key serine degradation pathways were removed, including serine deamination to pyruvate encoded by $s d a A, s d a B$ and $t d c G$ and serine conversion to glycine encoded by $g l y A$. The removal of glyA was shown to be crucial to enhance serine production. In C. glutamicum, a reduction of glyA expression (Peters-Wendisch et al., 2005) or activity by limiting the cofactor addition was found to enhance production. This method was later used to as a more successful strategy for accumulating $345 \mathrm{mM}$ Serine (Stolz et al., 2007). Construction of a glyA knock out has not been demonstrated in C. glutamicum, but was recently achieved in E. coli (Mundhada et al., 2016). However, the toxicity of serine significantly hampered the production. A serine tolerant strain, achieved by UV mutagenesis showed increased titer and growth fitness during fed batch fermentation, thus establishing a direct correlation between serine tolerance and production. Genome sequencing of the tolerant strain showed the deletion of $2855 \mathrm{bp}$, encoding a periplasmic protein involved in cell adhesion (ompX), osmoregulation $(o p g E)$, a threonine exporter $(r h t A)$ and a regulatory protein with unknown function ( $r y b A)$ (Mundhada et al., 2016).

L-serine has been reported to hamper a number of different cellular processes. Serine accumulation has been reported to inhibit cell division (Zhang and Newman, 2008) and peptidoglycan synthesis (Zhang et al., 2010). Serine could also potentially be converted to acrylates and other reactive intermediates (de Lorenzo et al., 2015) thereby affecting cell viability. Serine is also known to inhibit homoserine dehydrogenase (ThrA), which is involved in branched chain amino acid biosynthesis (Costrejean and Truffa-Bachi, 1977; Hama et al., 1990). As a result, threonine has been supplemented to the media in previous serine production experiments (Mundhada et al., 2016). To overcome these cellular bottlenecks, an approach such as Adaptive Laboratory Evolution (ALE) could be beneficial.

ALE is increasingly being used to enhance tolerance of cells towards inhibiting environmental conditions (Arense et al., 2010; Wang and Church, 2011), metabolites (Kwon et al., 2011) and oxygen limited conditions (Guadalupe-Medina et al., 2014). ALE has also been successfully applied for latent pathway activation (Portnoy et al., 2011) and for enhanced production of desired compound (Reyes et al., 2014). Several reviews have appeared on the ALE process (Conrad et al., 2011; Dragosits and Mattanovich, 2013). Although tolerance towards L-serine was previously achieved using random mutagenesis and selection (Mundhada et al., 2016), tolerance towards higher concentrations is still required for large scale serine production using E. coli. 
In this study, a serine sensitive production strain was subjected to ALE under increasing concentrations of Lserine. The evolved strains were genome sequenced and the effect of key mutations was investigated by reintroducing them into the parental strain. Among others, mutations in thrA revealed a potential serine binding site that is important for tolerance. The evolved strains showed significantly improved L-serine production during fed batch fermentation.

\section{Materials and Methods}

\subsection{Strains, reagents and equipment}

All chemicals were of analytical grade and ordered from Sigma-Aldrich (Taufkirchen, Germany). All strains and plasmids used in this study are summarized in Table S1. Strain Q1 carrying deletions of sdaA, sdaB, $t d c G$ and $g l y A$ was constructed in a previous study (Mundhada et al., 2016) and used as the parental strain for the ALE experiment. DNA oligonucleotides used for reverse engineering were ordered from Integrated DNA Technologies (Leuven, Belgium). Sequences and applications are listed in Tables S2, S3, S4 and S5 (Supplementary information). 2X YT media was used as a growth media for pre-culture and making electro competent cells. Antibiotics were added if required. ALE and tolerance studies were carried out and M9glycine media with varying concentration of L-serine. M9-glycine media contained $2 \mathrm{~g} / \mathrm{L}$ glucose, $2 \mathrm{mM}$ glycine, 1X M9 salts, $2 \mathrm{mM} \mathrm{MgSO}_{4}, 100 \mu \mathrm{M} \mathrm{CaCl}_{2}$ and $1 \mathrm{X}$ Trace elements and Wolfe's vitamin solution. M9-glycine media with $4 \mathrm{~g} / \mathrm{L}$ glucose was used for batch production experiments. Composition of $10 \mathrm{X}$ M9 salts and M9 trace elements was as described earlier (Mundhada et al., 2016). Growth profiles of strains were generated using a microtiter plate reader (Biotek, Winooski VT, USA). Phusion DNA polymerase and restriction enzymes were bought from Thermoscientific (Waltham, MA, USA). DNA and plasmid purifications were done by using Machery Nagel kits (Duren, German).

\subsection{Automated Adaptive Laboratory Evolution for L-Serine tolerance}

Five independent populations of the Q1 strain were adaptively evolved in M9 minimal media supplemented with $2 \mathrm{mM}$ glycine and increasing concentrations of the amino acid L-serine at $37{ }^{\circ} \mathrm{C}$ for a period of 45 days. The adaptive laboratory evolution experiment was carried out using an automated system, which enabled the propagation of evolving populations over the course of many days while monitoring their growth rates. Prior to the start of the experiment, the system filled the required tubes with $25 \mathrm{~mL}$ of culture media and kept them at $37{ }^{\circ} \mathrm{C}$ in a heat block. Controlled aeration was obtained using magnetic tumble stirrers placed inside the tubes and spinning at 1,800 rpm. At the start of the experiment, a single colony was grown overnight in one of the tubes placed inside the system, and $100 \mu \mathrm{L}$ aliquots were used by the robotics platform to inoculate five independent tubes. As the bacteria grew, the automated system performed multiple OD measurements at $600 \mathrm{~nm}$ for each flask. Growth rates were automatically calculated by taking the slope of a least-square linear regression line fit to the logarithm of the OD measurements. Once reaching a target OD600 of $0.4,100 \mu \mathrm{L}$ of 


\section{ACCEPTED MANUSCRIPT}

culture was used to inoculate a new flask. This way, cultures were serially passed ( $\sim 2-3$ times per day at the beginning of the experiment) to tubes with fresh media after reaching a targeted cell density such that stationary phase was never reached. The populations were initially incubated with $3 \mathrm{~g} / \mathrm{L}$ L-serine, after the desired growth rate was reached; cultures were supplemented with $6 \mathrm{~g} / \mathrm{L}$ of $\mathrm{L}$-serine. When populations achieved a stable phenotype (i.e. growth rate), the L-serine concentration was increased to $12 \mathrm{~g} / \mathrm{L}$. This process was repeated iteratively using $24,50,75$, and $100 \mathrm{~g} / \mathrm{L}$ of L-serine. Glycerol stocks were frozen approximately after every three days for genomic and phenotypic analysis.

\subsection{Genome and Amplicon sequencing}

Final populations were plated on LB-agar. Two clones from each of the evolved cultures including the parent strain were genome sequenced on MiSeq (Illumina Inc., San Diego, CA, USA) platform and analyzed as previously described (Mundhada et al., 2016) with the breseq analysis software (Deatherage and Barrick, 2014) using the NC_000913_3.gb as reference file (version 3 of the K-12 MG1655 reference).

A duplications module was generated to compare multiple genomes to a baseline in order to find the ranges where amplification in the compared genome differs from that of the baseline (i.e., Q1 parental strain). Genes corresponding to these ranges of differing amplifications, termed, 'duplications', were also found. To determine a duplicated region, each .bam file from breseq (Deatherage and Barrick, 2014) output was first parsed for the read depth over the entire genome to give an 'average read depth' and 'standard deviation'. The amplifications were then calculated by finding the ranges at which the read depth is higher than a threshold of 1.0 standard deviation above the average. These ranges were condensed by combining any ranges that were within 10 nucleotides of each other. Any leftover ranges under 300 nucleotides long were filtered out (as breseq likely calls these). The remaining ranges of amplifications were compared against a baseline (i.e., reference) genome to filter out known baseline repeat sequence / amplification regions (e.g., mobile elements). The output of this process was used to identify the duplications. Genbank reference files were then parsed to find genes contained within in the duplications.

Based on the sequencing results, key point mutations were reverse engineered using either the cat-sacB system (Sun et al., 2008) or an multiplex genome engineering (MAGE) approach as described below. The amplicon of targeted loci in MAGE were sequenced using a MiSeq instrument (Ilumina) and analyzed as previously described (Lennen et al., 2016).

\subsection{Reverse engineering of key mutations found in serine tolerant strains}

Mutations identified in thrA were introduced into the genome of the Q1(DE3) strain using a positivenegative $c a t-s a c B$ based selection system. The $t h r A$ alleles were amplified from respective ALE strains using thrA_gF and thrA_gR (Table S2, Supplementary information), while cat-sacB system targeting thrA was 
amplified using thrA_catsacB_F and R respectively (Table S2) and introduced in the genome of Q1(DE3) transformed with the pKD46 plasmid. Transformants were selected on LB-chloramphenicol plates. Later thrA alleles were individually transformed and selected on LB-sucrose plates (15\% sucrose, no $\mathrm{NaCl}$ ). $2 \mathrm{mM}$ glycine was supplemented in all the above media and plates.

Q1(DE3) thrA $S 357 R$ was used as a parental strain for multiplex genome engineering using plasmid pMA1 (Lennen et al., 2016). Colonies were cultured overnight in TY-amp-gly media at $37^{\circ} \mathrm{C}$ and re-inoculated in $25 \mathrm{ml}$ fresh TY-gly media the following day. After reaching an $\mathrm{OD}$ of 0.4 at $37^{\circ} \mathrm{C}$, arabinose was added to a final concentration of $0.2 \%$. Cells were re-incubated at $37{ }^{\circ} \mathrm{C}$ and $250 \mathrm{rpm}$ for additional $15 \mathrm{~min}$ and were then made electro-competent by washing the cells twice with $10 \%$ ice cold glycerol. Final volume of electro competent cells was adjusted to $200 \mu \mathrm{L}$ using $10 \%$ glycerol. The oligonucleotides targeting the loci $\operatorname{lrp}$ D143G, eno V164L, argP Q132K, cycA 1220V, pykF E250*, rpoB P520L, yojID334H, rho R87L were designed based on guidelines suggested by (Wang and Church, 2011) and are listed in supplementary Table S3. All primers were pooled and adjusted to a final concentration of $10 \mathrm{pmol} / \mu \mathrm{L}$. To $50 \mu \mathrm{l}$ of cells, $1 \mu \mathrm{L}$ of this mix was added and transformed by electroporation. The transformed cells were directly added to $25 \mathrm{ml}$ TY-gly media and incubated at $37{ }^{\circ} \mathrm{C}, 250 \mathrm{rpm}$ for two hours to reach OD of 0.4 , followed by arabinose induction and transformation ( $50 \mu \mathrm{L}$ from $250 \mu \mathrm{L}$ of electro competent cells) as described above for a subsequent round of multiplex engineering. After the sixth round of electroporation, cells were incubated overnight with no selection pressure. The resulting mutant library was subsequently grown in M9-glycine media with and without $12.5 \mathrm{~g} / \mathrm{L}$ L-serine. The frequency of individual mutants was determined by subjecting the culture for amplicon generation using primers listed in Table S4 followed by amplicon sequencing of targeted loci in three populations as mentioned before.

Two key mutations $\operatorname{lrp}$ D143G and rho R87L were introduced in Q1(DE3) thrA S357R strain separately using the CRMAGE system (Ronda et al., 2016). The pMAZSK plasmids containing gRNA targeting wild type lrp and rho was constructed using primers shown in Table S5. Forward and reverse gRNA primers (Table S5) each containing 11 and 9 bp of flanking gRNA sequence respectively were used to amplify the plasmid backbone by using Phusion polymerase in a two-step PCR with annealing temperature of $50^{\circ} \mathrm{C}$ for 5 cycle and $55^{\circ} \mathrm{C}$ for 20 cycles. The PCR product was subsequently phosphorylated with polynucleotide kinase, ligated with T4 DNA ligase and transformed. The isolated plasmids were used for the CRMAGE protocol (Ronda et al., 2016), and the resulting mutants were sequenced for targeted loci (by gF and gR primers listed in Table S5) and subjected for growth profiling studies.

\subsection{Modeling and docking studies of Homoserine dehydrogenase}

The ThrA homology model was constructed using the SWISS-MODEL server (Arnold et al., 2006). The crystal structure of ThrA from Methanococcus jannaschii (Faehnle et al., 2006) (PDB ID 2HMF) was used as a template for model construction. Coordinates for L-serine was obtained from PDBeChem. Docking 


\section{ACCEPTED MANUSCRIPT}

studies on the ThrA dimer model was performed using Autodock 4.2 (Morris et al., 2009) and structure visualization was carried out using Pymol (http://www.expasy.org/spdbv/).

\subsection{DE3 integration and batch fermentations of ALE strains for serine production}

Lambda DE3 lysogenization kit (Millipore, Damstadt Germany) was used for DE3 integration in the ALE strains. The kit, however, failed for the ALE-3 strains, and the DE3 cassette was therefore amplified from BL21-DE3 strain using USER primers (T7LacI_UF and UR) cloned in pOSIP-KO (St-Pierre et al., 2013) by amplifying the plasmid with pOSIP_UF and UR (Table S2, Supplementary information) for genome integration followed by loop out of integration cassette with pE-FLP. The plasmids for overexpression of the serine pathway (pCDFDuet-1-serAmut-serC and pACYCDuet-1-serB) (Mundhada et al., 2016) were transformed in the ALE-3-2(DE3), ALE-4-2(DE3) and ALE-5(DE3) strains respectively. The batch production of serine in ALE strains was similar to the protocol described previously with the exception that 4 $\mathrm{g} / \mathrm{L}$ of glucose was used and no threonine was added to the media. $80 \mu \mathrm{M}$ of IPTG was determined to be optimum for induction and this was used for induction of serine synthesis pathway. The concentration of glycine remained $2 \mathrm{mM}$.

\subsection{Serine production using fed batch fermentation}

Serine production was investigated during fed batch fermentation in $1 \mathrm{~L}$ fermenters (Sartorius, Gottingen, Germany). The media and feed composition of the Q1(DE3) and Q1-thrAS357R(DE3) were as previously described (Mundhada et al., 2016) with the exception that no threonine was added to the media and feed. For ALE-5(DE3) fermentation, the ammonium sulfate concentration was doubled in the batch media (10 g/L) and $1 \mathrm{X}$ vitamin solution was added. $250 \mathrm{~g}$ of feed contained, $600 \mathrm{~g} / \mathrm{L}$ glucose, $40 \mathrm{~g} / \mathrm{L}$ ammonium sulfate, 6 $\mathrm{g} / \mathrm{L}$ glycine, $2.5 \mathrm{~g} / \mathrm{L}$ of $\mathrm{MgSO}_{4} * 7 \mathrm{H}_{2} \mathrm{O}$ and $5 \mathrm{~g} / \mathrm{L}$ of $\mathrm{KH}_{2} \mathrm{PO}_{4}, 1 \mathrm{X}$ trace elements $50 \mathrm{mg} / \mathrm{L}$ spectinomycin and $25 \mathrm{mg} / \mathrm{L}$ chloramphenicol and $80 \mu \mathrm{M}$ of IPTG. An overnight culture was used to inoculate $500 \mathrm{ml}$ media in a 1:50 inoculum ratio. The cultures were allowed to grow to reach mid logarithmic phase in the fermenter at $37^{\circ} \mathrm{C}, 1000 \mathrm{rpm}$ baffle speed and $20 \%$ air saturation. Production was induced at late log phase (O.D. 8.5 to 9.5) by the addition of $40 \mu \mathrm{M}$ IPTG for Q1(DE3) and Q1 thrAS357R(DE3) strains and $80 \mu \mathrm{M}$ IPTG for ALE-5(DE3). Post induction, a feed of $6 \mathrm{~g} / \mathrm{h}$ was started; feed rate was tuned to between $4 \mathrm{~g} / \mathrm{h}$ or $8 \mathrm{~g} / \mathrm{h}$ based on glucose accumulation during fermentation. Samples were taken at regular intervals and were subjected to HPLC and LCMS analysis as mentioned before (Mundhada et al., 2016). Additionally, hydroxyglutarate was also analyzed on LCMS by monitoring the quantitative transition $147 \rightarrow 129$ and the qualitative transition was $147 \rightarrow 85$. 


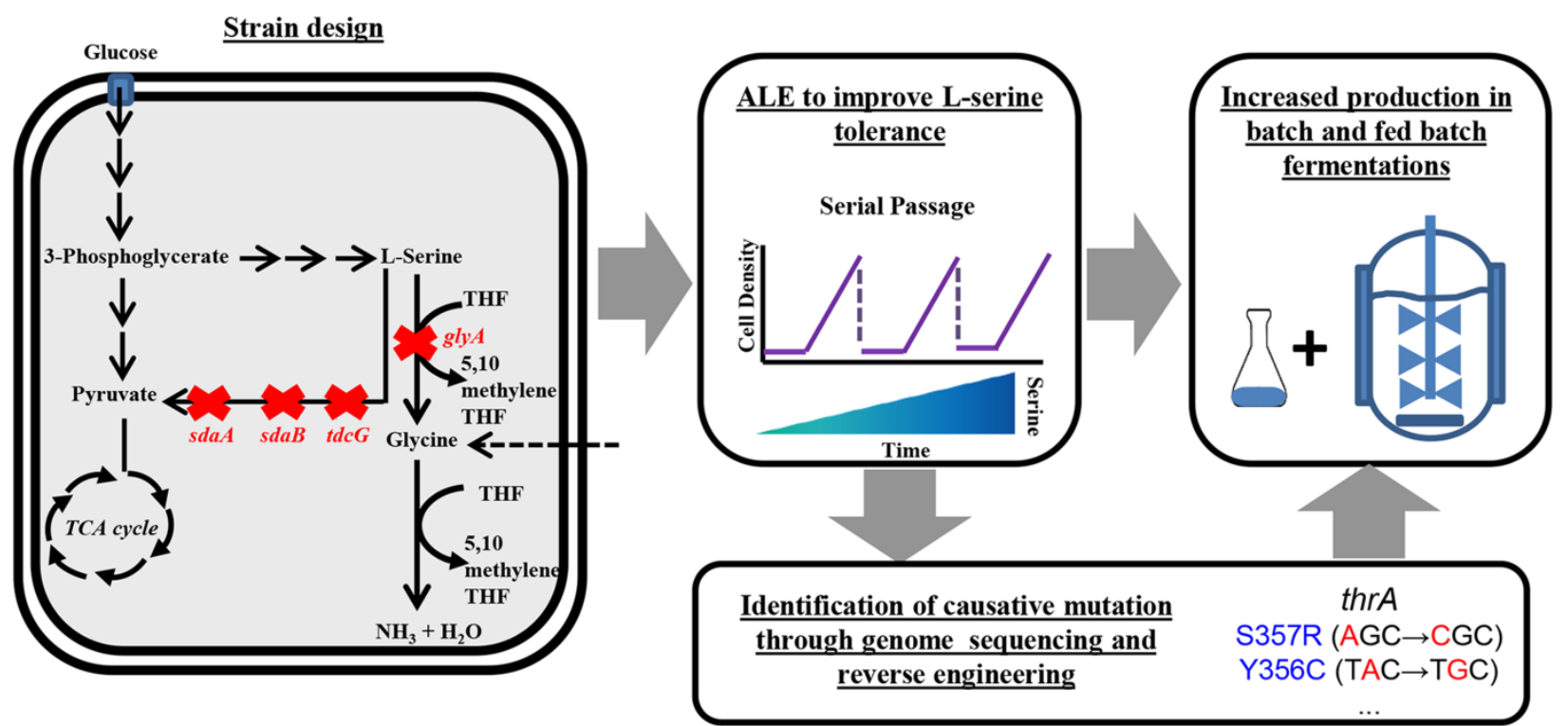

Figure 1: Schematic representation of the study performed to increase serine tolerance and production. A quadruple knock out strain (Q1) lacking key serine conversion pathways was subjected to ALE. Evolution was carried out such that cells were kept in the exponential growth phase. Strains isolated from the final evolved populations were subjected to whole genome sequencing and were also checked for L-serine production. Effects of key mutations observed in ALE populations were investigated using reverse engineering, combining MAGE and amplicon sequencing. Finally, the best performing strain identified under batch conditions was used for fed-batch production.

\section{Results and discussion}

\subsection{Adaptive evolution for L-serine tolerance}

In a recent study, high yield serine production in $E$. coli was achieved by removal of two key serine degradation pathways which constitute three L-serine deaminases ( $s d a A, s d a B, t d c G)$ as well as serine hydroxymethyl transferase ( $g l y A)$ catalyzing the conversion of serine to glycine (Mundhada et al., 2016). The resulting quadruple deletion strain is subsequently referred to as strain Q1. Though the Q1 strain showed a high serine yield during batch fermentations ( $0.42 \mathrm{~g} / \mathrm{g}$ glucose $)$, higher serine titers could not be achieved in fed batch fermentations due to sensitivity of this strain towards L-serine (Mundhada et al., 2016). In order to increase tolerance towards L-serine of the Q1 strain, we have here implemented ALE. The overall schematic of the process is shown in Figure 1. Five populations were evolved in parallel and L-serine concentration was increased gradually $(3,12,24,50,75$ and $100 \mathrm{~g} / \mathrm{L})$ during 45 days of evolution (Figure 2). The cells were continuously transferred into fresh media during the exponential growth phase. The concentration of Lserine was doubled when a desired growth rate was observed consistently for approximately 2-3 passages. For serine concentrations up to $24 \mathrm{~g} / \mathrm{L}$, a growth rate of 0.5 to 0.6 per hour was selected, while for higher 
concentrations ( $50 \mathrm{~g} / \mathrm{L}$ and above) the target was lowered to 0.2 to 0.4 per hour depending the population. The ALE profile of one of the population (ALE-5) is shown in Figure 2, while profiles for other key populations are shown in Figure S1 (Supplementary information).

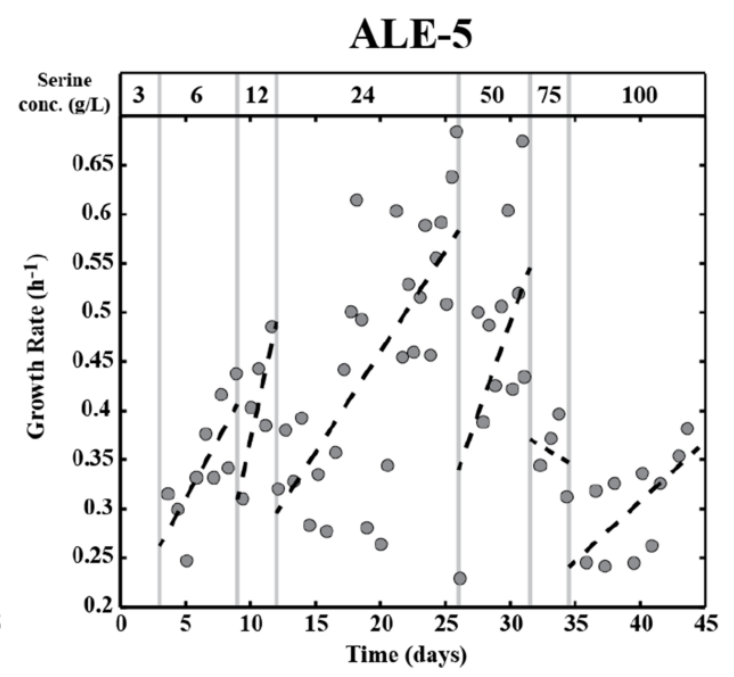

Figure 2: Adaptive evolution of serine tolerance. A plot of the growth rate as a function of time for one of the ALE populations (ALE-5) cultured under increasing amounts of L-serine during the experiment. The graph shows the measured growth rate for a representative culture during the ALE experiment. Each circle (gray) represents the growth rate from an individual culture and the dashed black 'regression' line is a linear fit for the growth rate over time for a given time period where the L-serine concentration was kept constant (concentration shown at top of the plot). Growth rates of some flasks during the experiment were not recorded. The vertical gray lines indicate the time points where the serine concentration was increased to increase the selection pressure. Clones were harvested at the end of the evolution for screening and resequencing. An overall trend of increasing growth rate over time was observed for most time periods where the concentration of L-serine was kept constant, indicating the populations were acquiring mutations that were beneficial to L-serine stress.

As shown by the regression lines in Figure 2 and Figure S1, overall increasing growth rates were observed during the evolution, indicating that strains were adapting towards the L-serine stress. At the end of the experiment, two colonies from each population were isolated, and the growth rates were determined for all strains in the presence of varying concentrations of L-serine $(0,12.5,25$ and $50 \mathrm{~g} / \mathrm{L})$. Both isolated clones of population ALE-4 and ALE-5 showed almost identical growth profiles. The ALE 3-2 clone showed much higher tolerance than ALE-3-1 (Supplementary Figure S2). The average growth rates of the evolved endpoint strains were compared to the parental Q1 strain, a serine tolerance strain generated through UV mutagenesis, the Q3 strain (Mundhada et al., 2016), as well as the K-12 MG1655 wild type strain. The growth rates of the most tolerant ALE strain (ALE-5) was higher than even the K-12 MG1655 strain at all serine concentrations. 
A clear difference was observed at $50 \mathrm{~g} / \mathrm{L}$ serine, where growth rate of ALE-5 endpoint strain was approximately 7.5 -fold higher than that of the K-12 MG1655 wild type strain. Similarly, the ALE-3-2 showed a 5.8-fold while ALE-4-2 had an approximately 4-fold higher growth rate (Figure 3A). The previously reported L-serine tolerant UV mutant showed marginal growth at $50 \mathrm{~g} / \mathrm{L}$ serine (Figure 3B) and thus the growth rate difference was between 17 to 34 -fold between ALE generated strains as compared to the Q3 strain (Figure 3A). Even at $25 \mathrm{~g} / \mathrm{L}$ serine, the ALE generated strains had a 1.5 to 2 -fold higher growth rate than the UV mutagenesis Q3 strain. The difference in growth rates attained by ALE strains in the ALE robotics and in microtiter plate experiments is potentially due to the lower degree of aeration in the microtiter plate reader.

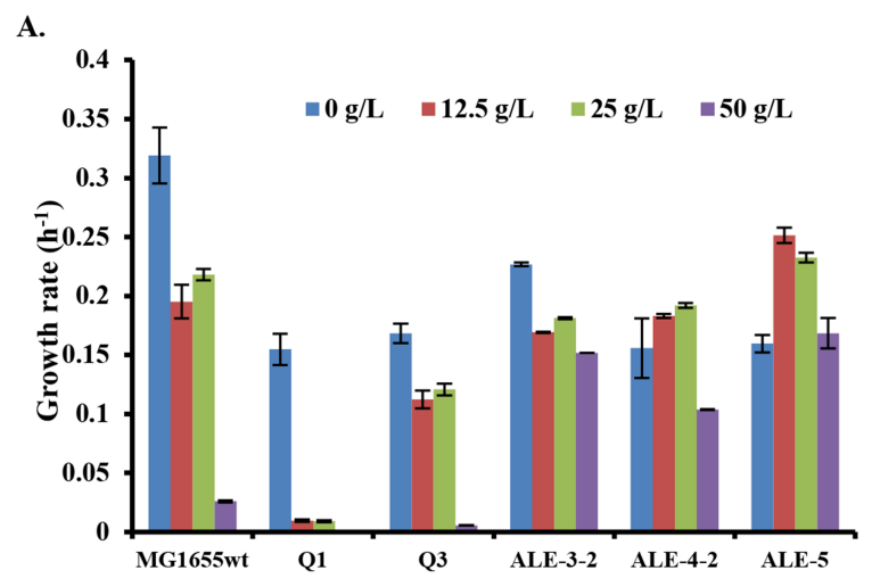

B.

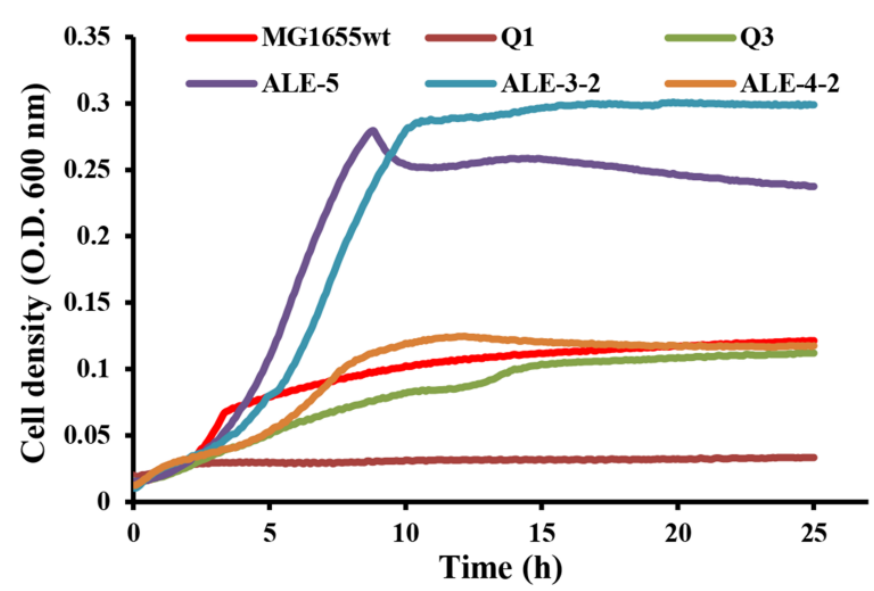

Figure 3: Growth rates and growth curves of ALE clones at varying L-serine concentrations. A. Comparison of growth rates of the K-12 MG1655 strain, the parental Q1 strain, a serine tolerant UV generated mutant Q3, and the ALE-3, ALE-4 and ALE-5 strains in M9-glycine minimal media supplemented with varying concentrations of serine. B. Corresponding growth profiles of the strains in the presence of 50 g/L L-serine.

\subsection{Genome sequencing of serine tolerant mutants}


Whole genome sequencing was utilized to determine the mutations fixed in the evolved strains. Genome sequencing of two clones from each of the ALE populations revealed that strains isolated from ALE-1 and ALE-2 were hypermutator strains carrying a very large number of mutations (approximately 100) and possessed mutations in the mutT ( $\Delta 1 \mathrm{bp}$ coding (8/390 nt)) and mutS (Y501D) mismatch repair genes. These strains were therefore not included in the subsequent analysis. The mutations found in the parental Q1 strain and each of the two strains isolated from ALE-3, 4 and 5 are shown in Table 1. Both ALE-5 clones were genetically identical and are therefore referred to as ALE-5, whereas ALE- 4 clones differed by 1 mutation. Although ALE 3-1 and ALE 3-2 differed only by a three point mutations, a gene duplication was observed in ALE 3-2 as described later. Mutations in the homoserine dehydrogenase (thrA) were observed in all three strains indicating the importance of this adaptation. Furthermore, mutations common between more than one strain included changes in promoter or coding regions of $r h o, \operatorname{lrp}$ and sRNA $g c v B$. Additionally, mutations were observed in the coding region of $r p o B, y o j I, c y c A, p y k F$ and number of other genes (Table 1). In order to identify the mutations causing the increased tolerance, a selected number of mutations were introduced into the parental strains as described below.

\subsection{Reverse engineering of thrA and other key mutations}

Mutations in thrA were identified in all three endpoint strains of ALE-3, 4 and 5, and were therefore expected to be important for serine tolerance. All three thrA mutations (Y356C, S357R and S359R) were therefore introduced separately into the parental Q1 (DE3) strain using the cat-sacB system (Sun et al., 2008). Growth profiles were performed for each of the generated strains at different serine concentrations (6.25 and 12.5), and all three alleles had a 10-30\% increased growth rate as compared to the Q1(DE3) strain at $6.25 \mathrm{~g} / \mathrm{L}$ of L-serine (Figure $4 \mathrm{~A}$ ). The growth rate of the thrA mutants was 3-fold to 3.5-fold higher at 12.5 $\mathrm{g} / \mathrm{L}$ of serine.

In order to identify which of the additional mutations found during the ALE experiment that confer tolerance towards L-serine and/or increased fitness in minimal media, a novel experimental approach that combines MAGE with amplicon sequencing of the targeted MAGE loci was implemented. Using MAGE, a set of mutations can be introduced into a strain and the resulting population can be enriched for the desired phenotype by growing the library with or without the growth inhibiting compound or condition. The beneficial mutations can subsequently be analyzed using next generation amplicon sequencing of the targeted loci.

Apart from mutations in thrA, other common mutations found the various ALE strains include changes in promoter or coding regions of rho, $\operatorname{lrp}$ and sRNA $g c v B$. As efficient MAGE recombineering requires multiple nucleotide changes, silent mutations are preferably included in the MAGE oligos (Wang and 


\section{ACCEPTED MANUSCRIPT}

Church, 2011). Since this is not possible for mutations in the sRNA, $g c v B$, and the promoters of rho and lrp, we chose to focus on coding mutations of rho and lrp. Of the other individual genetic changes shown in table 1, mutations in the coding region of $r p o B, y o j I, c y c A, p y k F$, eno, and $\arg P$ were selected based on predicted functions as described below and introduced into the Q1 (DE3) ThrA S357R strain.

The resulting random mutant library containing the selected mutations was grown in medium offering different selection pressures. Since the ALE experiment was carried out in minimal media containing serine, causative mutations were expected to both enhance fitness for minimal media and/or for serine tolerance. The frequency of the various mutations was determined using amplicon deep sequencing before and after selection for growth in minimal media with or without serine supplementation, thereby revealing the causative mutations. After six rounds of MAGE, the resulting library of mutants was grown in M9-glycine media with and without the supplementation of $12.5 \mathrm{~g} / \mathrm{L}$ L-serine. The original library was additionally grown in rich $2 \mathrm{xYT}$ media lacking any selection pressure as a reference. After overnight incubation, the mutated loci were PCR amplified from the three populations and subjected to next generation sequencing. This experiment enabled the determination of the enrichment of cells carrying various mutations in M9glycine media with and without L-serine as described below.

The MAGE-amplicon analysis revealed a 263-fold enrichment of the Lrp D143G mutation in the presence of serine (Figure 4B). Mutations in $\operatorname{lrp}$ itself or a one base pair change in its promoter region was found in both the ALE-4 and ALE-5 strains. Lrp is a global regulator that affects approximately $10 \%$ of genes of genome (Tani et al., 2002), making it challenging to identify the specific mechanism of tolerance. It is, however, known that $l r p$ - mutants are significantly more susceptible to L-serine than the lrp+ strain (Ambartsoumian et al., 1994) and the mutation in the promoter region of $\operatorname{lrp}$ may potentially enhance the expression of the gene. It is possible that the overexpression or D143G mutant of Lrp could further enhance the tolerance conferred by this global regulator at high serine concentrations. This was therefore further investigated by introducing the D143G mutation in Lrp on the genome by using the CRMAGE system. As shown in Figure $4 \mathrm{C}$, the introduction of the D143G mutation in Lrp increases the growth rate by 2.2, 2.6 and 6.5-fold at 6.25, 12.5 and $25 \mathrm{~g} / \mathrm{L}$ serine respectively when compared to the Q1 (DE3) thrA S357R strain. 


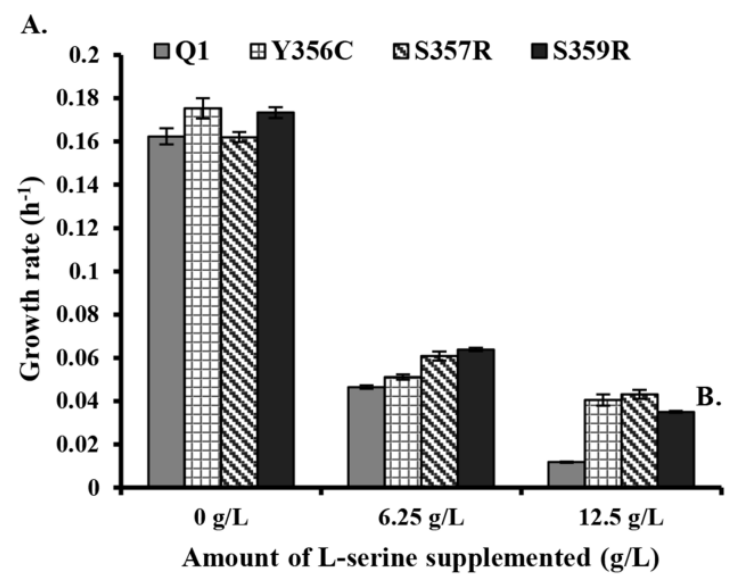

B.

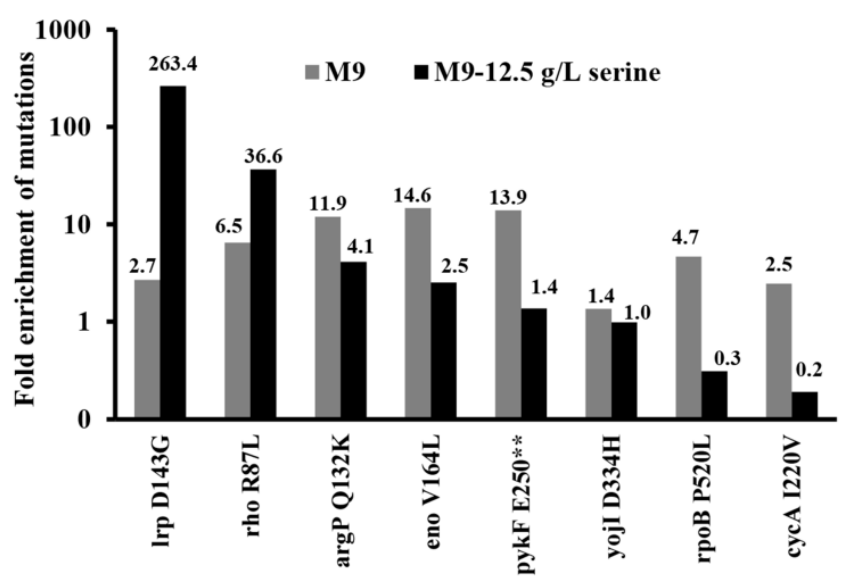

C.

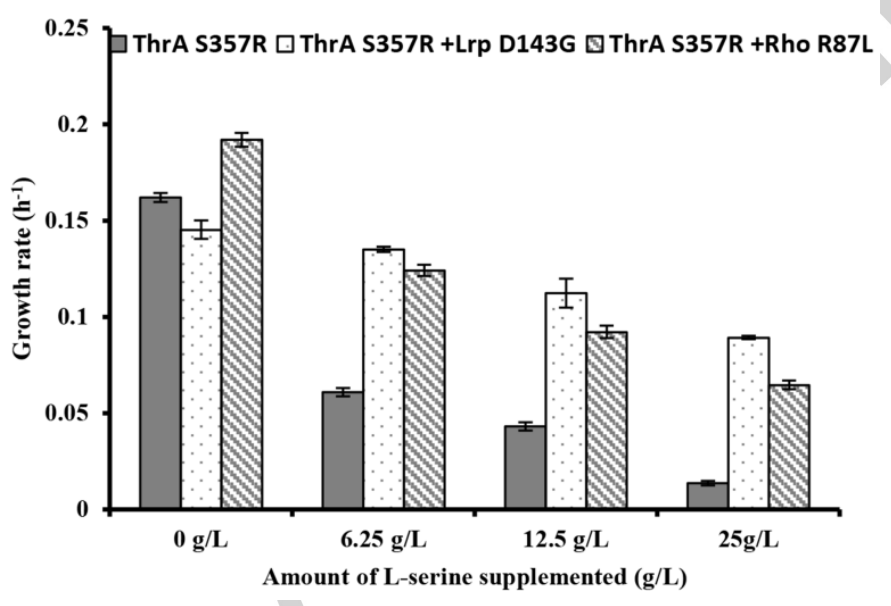

Figure 4. Reverse engineering of key mutations. A. Increase in growth rate of the parental strain at varying serine concentrations upon introduction of mutations in thrA. B. Selected mutations were introduced into the Q1 (DE3) ThrA S357R background using MAGE. Following selection for growth in minimal M9-glycine media or for tolerance towards $12.5 \mathrm{~g} / \mathrm{L}$ serine, amplicon sequencing was used to determine the fold enrichment of the targeted mutations. The control library was grown in rich media therefore offering no selection pressure. C. Increase in growth rate of Q1 (DE3) ThrA S357R strain at varying serine concentrations upon introduction of Lrp D143G or Rho R87L mutations. 


\section{ACCEPTED MANUSCRIPT}

Mutations in the transcription termination factor encoded by rho was another group of mutations commonly observed in the ALE populations. The ALE-5 strain has a R87L mutation, while the ALE-4 strain has a IS1 transposon insertion $19 \mathrm{bp}$ upstream of the rho gene. Therefore, the Rho R87L mutation was included in the reverse engineering experiment. The Rho R87L mutant showed the second highest enrichment (37-fold) in the presence of L-serine. Mutation in rho gene (L270M) has previously been observed in an ethanol tolerant strain (Haft et al., 2014), and Rho L270M was shown to have reduced activity and reduced the premature transcription terminations in the cell. It is possible that the R87L mutation and the insertion of transposon in the promoter region may also reduce the expression and activity of Rho. Interestingly, the Rho R87L mutation was also enriched by 6.5 -fold during growth in minimal media (Figure 4B). It is currently unclear why reduced premature transcription termination is a desirable property during growth in minimal media. The observed mutations in rho suggest that high concentrations of L-serine, like ethanol may affect the transcription machinery and was therefore further investigated by introducing rho R87L mutation on Q1(DE3) thrA S357R by CRMAGE system. As shown in Figure 4C., the introduction of R87L mutation in Rho increases the tolerance of the strain by $22.13,4.74$-fold at $6.25,12.5,25 \mathrm{~g} / \mathrm{L}$ of serine respectively. It can therefore be concluded that mutations in global regulators like Rho and Lrp play key roles to withstand serine toxicity and needs further elucidation.

Apart from the two mutations described above, most of the other mutations were shown to be more enriched during growth in minimal media than in the presence of L-serine (Figure 4B). The Q132K mutation of ArgP was found to be 12-fold enriched during growth in M9-glycine media. ArgP directly regulates DNA replication by binding to the oriC site and blocking the DNA opening by DnaA polymerase (Thony et al., 1991). A positive impact on serine tolerance of this mutation was also observed with a 4-fold increase in M9glycine medium supplemented with L-serine. Mutations in genes related to glycolysis were also commonly found in the evolved strains. A mutation in the enolase (EnoV 164L) and a truncation of pyruvate kinase I (PykF E250*) was observed in ALE-5 and ALE-3-2, respectively. The E250* mutation in PykF was enriched by 14 -fold during growth in minimal media, whereas hardly any effect was observed during growth in minimal medium containing L-serine. A similar enrichment (14.6-fold) was observed for the EnoV 164L mutation. This mutation also resulted in a 2.5 -fold enrichment in the presence of serine. Truncation at 250 amino acid of the pyruvate kinase would likely result in the loss of function of PykF as most of the active site residues are found after position 250 in the protein (Mattevi et al., 1995). Mutations in pykF (or in neighboring intergenic regions) have been identified in previous ALE studies selecting for increased growth rate in minimal media (LaCroix et al., 2015; Sandberg et al., 2014). It was found that mutations can result in decreased expression of PykF (Sandberg et al., 2014). Deletion of $p y k F$ has also been shown to down regulate glycolysis and upregulate the pentose phosphate pathway (Siddiquee et al., 2004). YojI has previously been shown to have a potential cysteine export function (Yamada et al., 2006) and the mutation 
YojI (D334H) was therefore included in the study. However, this point mutation did not show enrichment in either of the conditions.

A mutation in RpoB (P520L) was found to increase cell fitness in minimal medium (4.7-fold enrichment). However, the mutation was found to decrease the growth rate in the presence of L-serine (3-fold lower observed frequency) as shown in Figure 4B. Mutations in the RNA polymerase transcription unit rpoB, have previously been shown to be responsible for increasing fitness during growth in glucose containing minimal media (LaCroix et al., 2015; Sandberg et al., 2014). A similar effect was observed for the I220V mutation in the $c y c A$ gene, which encodes a transporter involved in the uptake of glycine, serine and alanine (Robbins and Oxender, 1973). The 2.5-fold enrichment of CycA I220V in minimal media potentially links the mutation to enhanced uptake of glycine, however simultaneous 5-fold depletion in serine supplemented media (Figure 4B) suggests that this mutation might also enhance the serine uptake, which is not desired in a serine producing strain.

Another common set of mutations identified in the ALE experiment included the sRNA $g c v B$, which is localized in the region between the glycine cleavage operon regulator, $g c v A$, and the lipoprotein encoded by $y g d L$. The sRNA $g c v B$ negatively regulates expression of the serine importers sst $T$ and $c y c A$ (Pulvermacher et al., 2009a; Pulvermacher et al., 2009b). It has also been shown to repress the global regulator lrp (Modi et al., 2011). The overexpression of $g c v B$ in minimal media containing L-serine has been shown to inhibit the growth of the bacteria (Modi et al., 2011). Therefore, a down-regulation or truncation of $g c v B$ will be expected to positively affect serine tolerance of the cell. Elucidation of the effect of transcription and truncation of $g c v B$ sRNA will require further detailed studies.

\subsection{Duplications identified in evolved strains}

Duplicated regions were identified in the evolved strains which can be linked to L-serine tolerance. Analysis of ALE-3 and ALE-5 strains showed that one of the ALE-3 strains (ALE-3-2) and both ALE-5 strains (ALE5-1 and 5-2) have clusters of genes duplicated, potentially more than twice (Table S6). In ALE-3-2, a cluster with a size of approximately $56 \mathrm{~kb}$, which includes genes from hipA to $f l x A$, was found to have a higher multiplicity as determined by the average read depth. Interestingly, a small part of this cluster which constitutes around $3.6 \mathrm{~kb}$ starting from the marA gene to $d g c Z$ was found to have a multiplicity significantly higher than two copies given the read depth in this region, suggesting that a nested duplication had occurred (Supplementary Figure S3A). Both ALE-5 clones showed an essentially identical duplication of a $9.7 \mathrm{~kb}$ region containing 14 open reading frames (Supplementary Figure S3B). Since the duplicated regions were different between the ALE3-2 and ALE-5 strains, it was concluded that these duplications were the result of two independent events. The duplications were not found in the starting strain or in the other evolved clones (Figure S3C). Since the duplication in ALE-3-2 and ALE-5 share similar sets of genes, it is likely that they may be involved in L-serine tolerance. This was supported by the fact that the ALE-3-1 clone, which did not 
contain the duplication, was found to have a significantly lower L-serine tolerance when compared to the ALE-3-2 strain (Supplementary Figure S2).

The genes present in the amplified clusters encode the active transporters YdeA, EamA and YdeE as well as putative proteins with unknown functions, including YneJ and YneK. In a previous study we have shown that enhancing the serine export by overexpression of EamA significantly increases tolerance of the Q1 strain (Mundhada et al., 2016). It is highly likely that the duplication may be upregulating the expression of eamA. The cluster additionally contains the marRAB operon which participates in regulating more than 80 genes involved in resistance to multiple antibiotic, heavy metals and organic compounds such as salicylate (Ariza et al., 1994). Furthermore, the cluster contains stress induced cell membrane proteins such as YneM (Hemm et al., 2010), YdeI (Lee et al., 2010) as well as the sRNA MgrR (Moon and Gottesman, 2009). The multiplication of this genomic region is therefore likely contributing to resistance towards L-serine though increased exporter activity as well as osmotic tolerance.

\subsection{Structural modeling studies of L-serine inhibition of ThrA}

Since all the evolved strains carried mutations at three neighboring positions in ThrA, Y356, S357 and S359, we wanted to further investigate if these amino acid residues could be part of a potential L-serine binding site. Homoserine dehydrogenase is involved in branched amino acid synthesis, and it is known to be inhibited by L-serine (Costrejean and Truffa-Bachi, 1977; Hama et al., 1990). However, the mechanism and site of inhibition has not been identified. The E. coli ThrA is reported to consist of a dimer of dimers (Heck, 1972) but has yet to be crystallized. In order to study the localization of the adjacent mutations, a homology model of ThrA was therefore constructed based on the crystal structure of ThrA from Methanococcus (Faehnle et al., 2006), which was showing $37 \%$ identity at the protein level. The model shows interaction of two regulatory domains (Figure 5A), where each regulatory domain consists of ACT1 and ACT2 domains. The three mutated residues Y356, S357 and S359 are part of the third beta strand of the ACT1 domain (Figure 5A). Interestingly, they are localized in close proximity to the threonine inhibition site at S345 (Lee et al., 2003). Docking studies with L-serine revealed that the hydroxyl group of S357 forms a hydrogen bond with the ammonium terminus of L-serine while the hydroxyl group of S359 interacts with the hydroxyl group of L-serine thus stabilizing the interaction (Figure 5B). When S357 is mutated to arginine, the longer amine side chain leads to steric hindrance preventing serine from binding (Figure 5C). A similar effect on serine binding is expected for the S359R and Y356C substitutions. 


\section{ACCEPTED MANUSCRIPT}

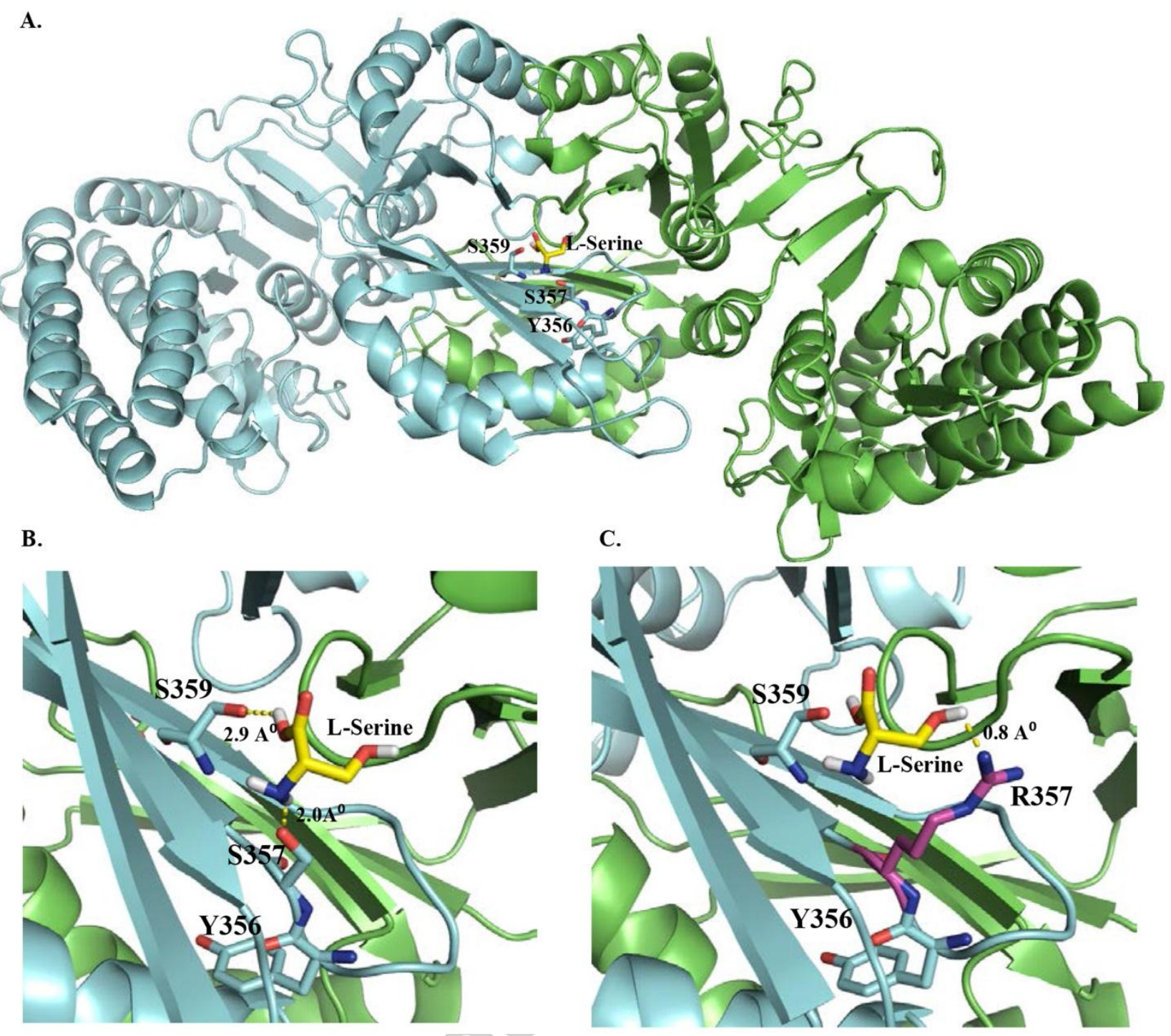

Figure 5 A. Modeling studies of homoserine dehydrogenase. A. Docking of L-serine on the dimer of the E. coli homoserine dehydrogenase (ThrA) homology model (residue 3 to 460) constructed using SWISS MODEL. B. Close view of the docking of L-serine on the dimer. L-serine (yellow) is stabilized by forming hydrogen bond with S357 and S359 leading to potential enzyme inhibition. C. Close view of the ThrA S357R mutant aligned to the wild type ThrA, which is docked with serine. The R357 mutation causes steric hindrance and likely prevents binding of L-serine.

\subsection{Mutations in thrA bypasses requirement of threonine supplementation}

In a previous study focusing on L-serine production in $E$. coli, threonine supplementation was found to be required to allow cells to grow at a higher rate and produce more serine (Mundhada et al., 2016). To investigate if mutations in thrA would eliminate the requirement for threonine supplementation, a serine production pathway was overexpressed in the Q1-thrA S357R (DE3) strain. Genes containing a feedback insensitive serAmut, as well as $\operatorname{ser} C$ and $\operatorname{ser} B$ were overexpressed from pET-Duet vectors (Mundhada et al., 
2016). Upon induction of the L-serine pathway and supplementation of threonine (in media and feed), the parental Q1 strain reached an OD of 8.5 and produced $8.3 \mathrm{~g} / \mathrm{L}$ serine (Mundhada et al., 2016). However, without threonine supplementation, the Q1 strain did not grow to an OD600 higher than 5.5 and the cell density dropped to an OD600 of 4 when the L-serine pathway expression was induced (Figure 6A). At the end of the fermentation, L-serine accumulation had only reached $3.7 \mathrm{~g} / \mathrm{L}$. The Q1(DE3) thrA S357R strain, however, showed no growth inhibition in the absence of threonine, and the final cell density was 3-fold higher when compared to that obtained from the Q1 strain supplemented with threonine. The L-serine production for the Q1(DE3) thrA S357R strain was $9 \mathrm{~g} / \mathrm{L}$, which was 2.5-fold higher than the Q1(DE3) strain under identical conditions.

\subsection{L-serine production during batch fermentation}

To evaluate the potential of the evolved strain for L-serine production under batch conditions, a serine production pathway containing a feedback insensitive serAmut, serC and $\operatorname{ser} B$ was overexpressed in the evolved endpoint strains using pET-Duet vectors as reported earlier (Mundhada et al., 2016). Supplementation of the media with threonine was omitted since all the tested strains contained mutations in thrA, making them independent of threonine addition. Upon induction (OD600 0.5 to 0.65), serine concentrations steadily increased in all ALE strains, however, the growth and productivity of ALE-5(DE3) was found to be the best (Figure 6B). Between 13-15 h after inoculation, the ALE-5 strain showed 2.6-fold higher L-serine titer when compared to ALE-4 and ALE-3, respectively (Figure 6B). The high titer was due to the high growth rate attained by ALE-5 under serine production conditions. The ALE-3-2 strain grew better than the ALE-4 strain and did not show decreased growth post induction. This observation matches well the observed tolerance of ALE strains, however, ALE-3-2 did not show higher titers than ALE-4-2. The ALE-5 strain was therefore used for L-serine production during fed batch fermentation. 
A.

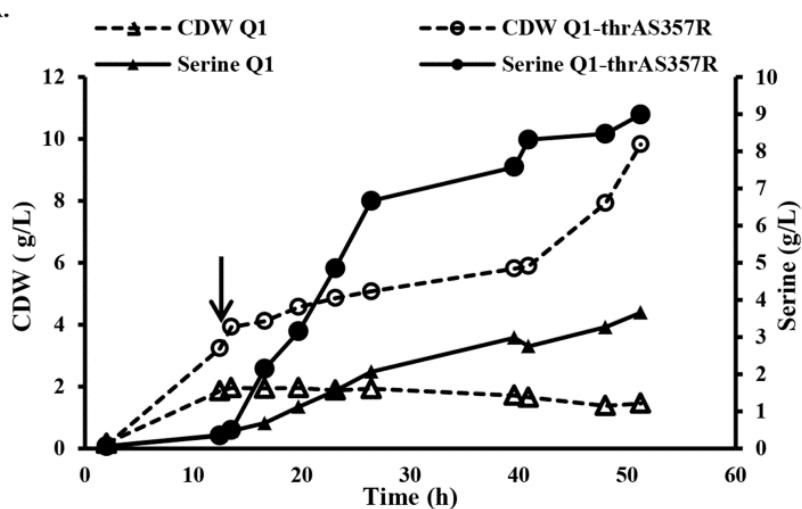

B.

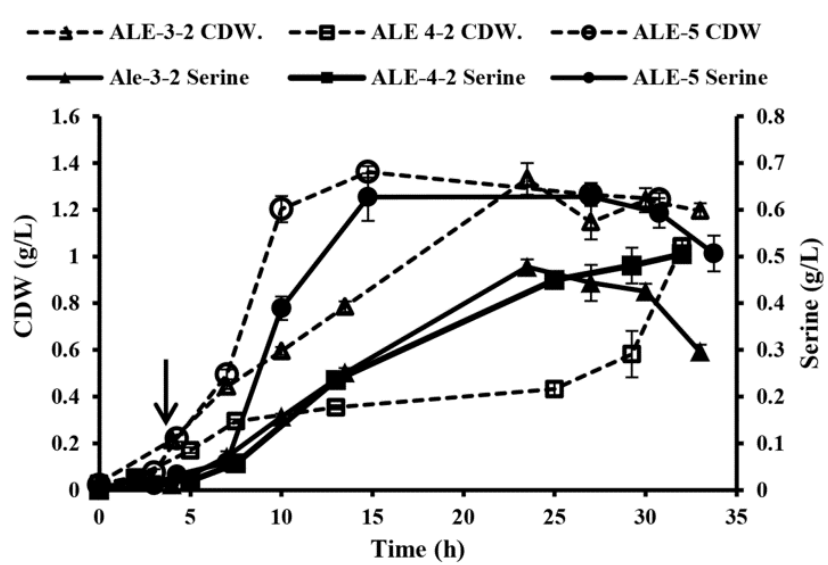

C.

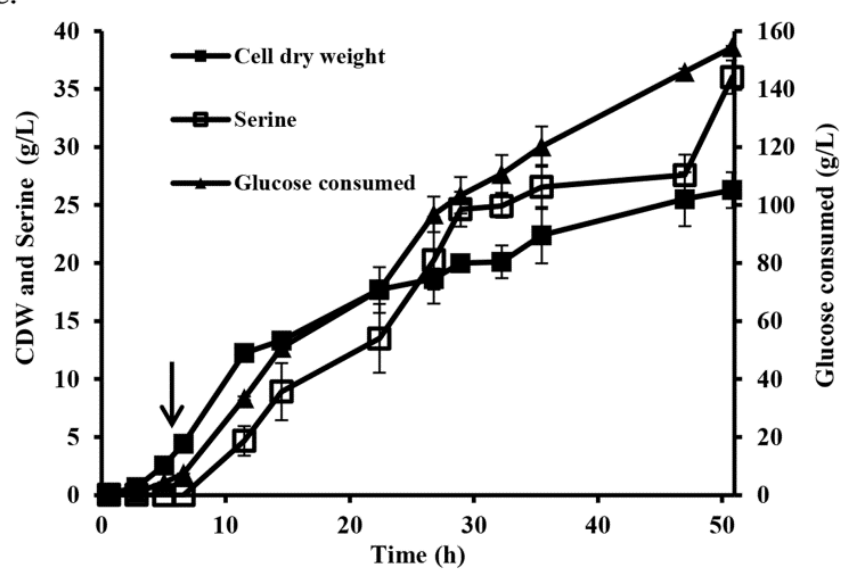

Figure 6: Serine production during batch and fed batch fermentations. A. Serine production and cell density of the Q1(DE3) and Q1-thrA S357R(DE3) strain during fed batch fermentation. B. Cell density and serine production achieved by the ALE-3, ALE-4 and ALE-5 strains containing plasmids pCDF-Duet1serAmut-serC and pACYC-serB. Error bars represent standard deviations calculated from triplicate experiments. C. Fed-batch serine production using ALE-5 strain. The graph shows serine production, cell dry weight (cdw) and glucose consumed as a function of time. The error bars represent variations from duplicate experiments. Arrows indicate the point of induction. 


\section{ACCEPTED MANUSCRIPT}

\subsection{Serine production during fed batch fermentation}

Since the serine production strains are glycine auxotrophs, we initially tested the effect of glycine supplementation (from $3 \mathrm{~g} / \mathrm{L}$ to $6 \mathrm{~g} / \mathrm{L}$ ) to the feed during the fed batch fermentation. Even though differences in cell growth and serine production were observed, the amount of glucose consumed by the strains was interestingly found to be the same (Figure S3). Since the highest serine production was observed when $6 \mathrm{~g} / \mathrm{L}$ of glycine was included in the feed, this concentration was used for further fermentation studies.

The serine pathway was induced by adding IPTG $(80 \mu \mathrm{M}$ final concentration) after the cells reached an optical density from 8 to 8.5 . L-serine production as well as the cell density increased steadily until $35 \mathrm{~h}$ of fermentation (Figure 6C). During entry into stationary phase, the rate of serine production was found to decrease, however, it increased later during stationary phase. A final titer of $37.3 \mathrm{~g} / \mathrm{L}$ was reached after 50 hours of fermentation, and no further accumulation was observed after this time point. This titer was more than 3 times higher than obtained in previous studies using E. coli as a production host (Mundhada et al., 2016). A total of $125 \mathrm{~g}$ glucose was consumed, resulting in a yield of $0.24 \mathrm{~g} / \mathrm{g}$. This yield is slightly lower than the $0.33 \mathrm{~g} / \mathrm{g}$ that was previously obtained for strain with the same production vector backbone. This may be caused by the increased tolerance of the ALE strain, which resulted in a 5.2-fold increased biomass accumulation. The increase in biomass also lead to an increase in productivity to $0.83 \mathrm{~g} / \mathrm{L} / \mathrm{h}$ post induction as compared to the $0.24 \mathrm{~g} / \mathrm{L} / \mathrm{h}$ previously obtained for the Q3 strain (Mundhada et al., 2016). The current strain has a potential multiplication of eamA, which has previously been shown to increase productivity. We therefore decided not to further overexpress this transporter. Along with a threefold increase in L-serine titer, an approximately threefold increase in accumulation of the byproduct $\alpha$-hydroxyglutarate $(12.1 \mathrm{~g} / \mathrm{L})$ was also observed. Hydroxyglutarate formation may be the result of the promiscuous activity of SerA on $\alpha$ ketoglutarate ( $\mathrm{Gu}$ et al., 2014; Zhao and Winkler, 1996). To further increase L-serine yield, it will be important to address the production of $\alpha$-hydroxyglutarate since it represents a significant carbon sink.

\section{Conclusion}

In this work, we describe the utility of adaptive laboratory evolution as a tool not just to study the underlying mechanism of toxicity and tolerance, but to obtain an evolved strain that enhances the production of Lserine. The most promising strain generated using this approach achieved a threefold higher titer compared to what has previously been achieved for E. coli (Mundhada et al., 2016). The value reported for the strain developed in the present study is close to highest titer and yield reported for any organism (Zhu et al., 2015). The mutations identified in thrA hints towards a mechanism of inhibition of the homoserine dehydrogenase, and they furthermore eliminated the requirement for threonine supplementation to the fermentation media. This will further help to reduce the cost of L-serine production. During the ALE process, the cells were found to evolve and fix individual mutations, which could be examined for causality using genome-scale engineering techniques. This resulted in a deeper insight into the underlying mechanisms, including the 


\section{ACCEPTED MANUSCRIPT}

potential site of inhibition of ThrA. Through enrichment and amplicon sequencing of populations engineered at desired loci, we were able to show that not all mutations were necessarily acquired to enhance the tolerance, but instead enhanced the fitness of the cells in minimal media. This approach for screening and differentiating causative mutations has to best of our knowledge not previously been applied. Interestingly, the most tolerant ALE mutants (ALE 3-2 and ALE 5-1 and 5-2) showed duplications of a set of gene clusters involved in serine export and osmotic tolerance. The duplication of segments of the genome is yet another means by which the cells can adapt to stressful conditions, such as high concentrations of L-serine. Additional key adaptations included perturbations of the genes encoding global regulators, lrp, rho and sRNA $g c v B$. To further investigate the role of these regulators, a systems level approach will be required. The performed experiments demonstrate the power of using ALE for rapidly improving production strains for industrial biotechnology.

\section{Acknowledgements}

The research was funded by The Novo Nordisk Foundation as well as Proof of Concept funding from the Technical University of Denmark. Authors are thankful to Inger Rosenstand and Yasin Dastan for their assistance with fed batch fermentations. We would also like to thank Dennis Gosting for his help with the sequencing analysis. The authors declare not to have any conflict of interest.

\section{References}

Ambartsoumian, G., D'Ari, R., Lin, R. T., Newman, E. B., 1994. Altered amino acid metabolism in lrp mutants of Escherichia coli K12 and their derivatives. Microbiology. 140 ( Pt 7), 1737-44.

Arense, P., Bernal, V., Iborra, J. L., Cánovas, M., 2010. Metabolic adaptation of Escherichia coli to longterm exposure to salt stress. Process Biochemistry. 45, 1459-1467.

Ariza, R. R., Cohen, S. P., Bachhawat, N., Levy, S. B., Demple, B., 1994. Repressor mutations in the marRAB operon that activate oxidative stress genes and multiple antibiotic resistance in Escherichia coli. Journal of bacteriology. 176, 143-8.

Arnold, K., Bordoli, L., Kopp, J., Schwede, T., 2006. The SWISS-MODEL workspace: a web-based environment for protein structure homology modelling. Bioinformatics. 22, 195-201.

Becker, J., Wittmann, C., 2012. Systems and synthetic metabolic engineering for amino acid production - the heartbeat of industrial strain development. Curr Opin Biotechnol. 23, 718-26.

Conrad, T. M., Lewis, N. E., Palsson, B. O., 2011. Microbial laboratory evolution in the era of genome-scale science. Molecular systems biology. 7, 509 .

Costrejean, J. M., Truffa-Bachi, P., 1977. Threonine-sensitive homoserine dehydrogenase and aspartokinase activities of Escherichia coli K12. Kinetic and spectroscopic effects upon binding of serine and threonine. The Journal of biological chemistry. 252, 5332-6.

de Lorenzo, V., Sekowska, A., Danchin, A., 2015. Chemical reactivity drives spatiotemporal organisation of bacterial metabolism. FEMS microbiology reviews. 39, 96-119.

Deatherage, D. E., Barrick, J. E., 2014. Identification of mutations in laboratory-evolved microbes from next-generation sequencing data using breseq. Methods in molecular biology. 1151, 165-88. 
Dragosits, M., Mattanovich, D., 2013. Adaptive laboratory evolution -- principles and applications for biotechnology. Microb Cell Fact. 12, 64.

Faehnle, C. R., Liu, X., Pavlovsky, A., Viola, R. E., 2006. The initial step in the archaeal aspartate biosynthetic pathway catalyzed by a monofunctional aspartokinase. Acta Crystallogr Sect F Struct Biol Cryst Commun. 62, 962-6.

Gu, P., Yang, F., Su, T., Li, F., Li, Y., Qi, Q., 2014. Construction of an L-serine producing Escherichia coli via metabolic engineering. Journal of industrial microbiology \& biotechnology. 41, 1443-50.

Guadalupe-Medina, V., Metz, B., Oud, B., van Der Graaf, C. M., Mans, R., Pronk, J. T., van Maris, A. J., 2014. Evolutionary engineering of a glycerol-3-phosphate dehydrogenase-negative, acetate-reducing Saccharomyces cerevisiae strain enables anaerobic growth at high glucose concentrations. Microbial biotechnology. 7, 44-53.

Haft, R. J., Keating, D. H., Schwaegler, T., Schwalbach, M. S., Vinokur, J., Tremaine, M., Peters, J. M., Kotlajich, M. V., Pohlmann, E. L., Ong, I. M., Grass, J. A., Kiley, P. J., Landick, R., 2014. Correcting direct effects of ethanol on translation and transcription machinery confers ethanol tolerance in bacteria. Proceedings of the National Academy of Sciences of the United States of America. 111, E2576-85.

Hama, H., Sumita, Y., Kakutani, Y., Tsuda, M., Tsuchiya, T., 1990. Target of serine inhibition in Escherichia coli. Biochemical and biophysical research communications. 168, 1211-6.

Heck, H., 1972. Threonine-sensitive aspartokinase--homoserine dehydrognease of Escherichia coli K 12 . Evidence for a cooperative tetramer. Biochemistry. 11, 4421-7.

Hemm, M. R., Paul, B. J., Miranda-Rios, J., Zhang, A., Soltanzad, N., Storz, G., 2010. Small stress response proteins in Escherichia coli: proteins missed by classical proteomic studies. Journal of bacteriology. 192, 46-58.

Kwon, Y. D., Kim, S., Lee, S. Y., Kim, P., 2011. Long-term continuous adaptation of Escherichia coli to high succinate stress and transcriptome analysis of the tolerant strain. J Biosci Bioeng. 111, 26-30.

LaCroix, R. A., Sandberg, T. E., O'Brien, E. J., Utrilla, J., Ebrahim, A., Guzman, G. I., Szubin, R., Palsson, B. O., Feist, A. M., 2015. Use of adaptive laboratory evolution to discover key mutations enabling rapid growth of Escherichia coli K-12 MG1655 on glucose minimal medium. Applied and environmental microbiology. 81, 17-30.

Lee, J., Hiibel, S. R., Reardon, K. F., Wood, T. K., 2010. Identification of stress-related proteins in Escherichia coli using the pollutant cis-dichloroethylene. J Appl Microbiol. 108, 2088-102.

Lee, J. H., Lee, D. E., Lee, B. U., Kim, H. S., 2003. Global analyses of transcriptomes and proteomes of a parent strain and an L-threonine-overproducing mutant strain. Journal of bacteriology. 185, 5442-51.

Lennen, R. M., Nilsson Wallin, A. I., Pedersen, M., Bonde, M., Luo, H., Herrgard, M. J., Sommer, M. O., 2016. Transient overexpression of DNA adenine methylase enables efficient and mobile genome engineering with reduced off-target effects. Nucleic Acids Res. 44, e36.

Leuchtenberger, W., Huthmacher, K., Drauz, K., 2005. Biotechnological production of amino acids and derivatives: current status and prospects. Applied microbiology and biotechnology. 69, 1-8.

Mattevi, A., Valentini, G., Rizzi, M., Speranza, M. L., Bolognesi, M., Coda, A., 1995. Crystal structure of Escherichia coli pyruvate kinase type I: molecular basis of the allosteric transition. Structure. 3, 72941.

Modi, S. R., Camacho, D. M., Kohanski, M. A., Walker, G. C., Collins, J. J., 2011. Functional characterization of bacterial sRNAs using a network biology approach. Proceedings of the National Academy of Sciences of the United States of America. 108, 15522-7.

Moon, K., Gottesman, S., 2009. A PhoQ/P-regulated small RNA regulates sensitivity of Escherichia coli to antimicrobial peptides. Molecular microbiology. 74, 1314-30.

Morris, G. M., Huey, R., Lindstrom, W., Sanner, M. F., Belew, R. K., Goodsell, D. S., Olson, A. J., 2009. AutoDock4 and AutoDockTools4: Automated docking with selective receptor flexibility. J Comput Chem. 30, 2785-91.

Mundhada, H., Schneider, K., Christensen, H. B., Nielsen, A. T., 2016. Engineering of high yield production of L-serine in Escherichia coli. Biotechnology and bioengineering. 113, 807-16. 
Pereira, B., Zhang, H., De Mey, M., Lim, C. G., Li, Z. J., Stephanopoulos, G., 2016. Engineering a novel biosynthetic pathway in Escherichia coli for production of renewable ethylene glycol. Biotechnology and bioengineering. 113, 376-83.

Peters-Wendisch, P., Stolz, M., Etterich, H., Kennerknecht, N., Sahm, H., Eggeling, L., 2005. Metabolic engineering of Corynebacterium glutamicum for L-serine production. Applied and environmental microbiology. 71, 7139-44.

Portnoy, V. A., Bezdan, D., Zengler, K., 2011. Adaptive laboratory evolution--harnessing the power of biology for metabolic engineering. Curr Opin Biotechnol. 22, 590-4.

Pulvermacher, S. C., Stauffer, L. T., Stauffer, G. V., 2009a. Role of the sRNA GcvB in regulation of cycA in Escherichia coli. Microbiology. 155, 106-14.

Pulvermacher, S. C., Stauffer, L. T., Stauffer, G. V., 2009b. The small RNA GcvB regulates sstT mRNA expression in Escherichia coli. Journal of bacteriology. 191, 238-48.

Reyes, L. H., Gomez, J. M., Kao, K. C., 2014. Improving carotenoids production in yeast via adaptive laboratory evolution. Metabolic engineering. 21, 26-33.

Robbins, J. C., Oxender, D. L., 1973. Transport systems for alanine, serine, and glycine in Escherichia coli K-12. Journal of bacteriology. 116, 12-8.

Ronda, C., Pedersen, L. E., Sommer, M. O., Nielsen, A. T., 2016. CRMAGE: CRISPR Optimized MAGE Recombineering. Scientific reports. 6, 19452.

Sandberg, T. E., Pedersen, M., LaCroix, R. A., Ebrahim, A., Bonde, M., Herrgard, M. J., Palsson, B. O., Sommer, M., Feist, A. M., 2014. Evolution of Escherichia coli to 42 degrees C and subsequent genetic engineering reveals adaptive mechanisms and novel mutations. Molecular biology and evolution. 31, 2647-62.

Siddiquee, K. A., Arauzo-Bravo, M. J., Shimizu, K., 2004. Effect of a pyruvate kinase (pykF-gene) knockout mutation on the control of gene expression and metabolic fluxes in Escherichia coli. FEMS Microbiol Lett. 235, 25-33.

St-Pierre, F., Cui, L., Priest, D. G., Endy, D., Dodd, I. B., Shearwin, K. E., 2013. One-step cloning and chromosomal integration of DNA. ACS Synth Biol. 2, 537-41.

Stolz, M., Peters-Wendisch, P., Etterich, H., Gerharz, T., Faurie, R., Sahm, H., Fersterra, H., Eggeling, L., 2007. Reduced folate supply as a key to enhanced L-serine production by Corynebacterium glutamicum. Applied and environmental microbiology. 73, 750-5.

Sun, W., Wang, S., Curtiss, R., 3rd, 2008. Highly efficient method for introducing successive multiple scarless gene deletions and markerless gene insertions into the Yersinia pestis chromosome. Applied and environmental microbiology. 74, 4241-5.

Tani, T. H., Khodursky, A., Blumenthal, R. M., Brown, P. O., Matthews, R. G., 2002. Adaptation to famine: a family of stationary-phase genes revealed by microarray analysis. Proceedings of the National Academy of Sciences of the United States of America. 99, 13471-6.

Thony, B., Hwang, D. S., Fradkin, L., Kornberg, A., 1991. iciA, an Escherichia coli gene encoding a specific inhibitor of chromosomal initiation of replication in vitro. Proceedings of the National Academy of Sciences of the United States of America. 88, 4066-70.

Wang, H. H., Church, G. M., 2011. Multiplexed genome engineering and genotyping methods applications for synthetic biology and metabolic engineering. Methods in enzymology. 498, 409-26.

Werpy, T., Petersen, G., Top Value Added Chemicals from Biomass: Results of Screening for Potential Candidates from Sugars and Synthesis Gas. 1, 1-76.

Yamada, S., Awano, N., Inubushi, K., Maeda, E., Nakamori, S., Nishino, K., Yamaguchi, A., Takagi, H., 2006. Effect of drug transporter genes on cysteine export and overproduction in Escherichia coli. Applied and environmental microbiology. 72, 4735-42.

Zhang, X., El-Hajj, Z. W., Newman, E., 2010. Deficiency in L-serine deaminase interferes with one-carbon metabolism and cell wall synthesis in Escherichia coli K-12. Journal of bacteriology. 192, 5515-25.

Zhang, X., Newman, E., 2008. Deficiency in 1-serine deaminase results in abnormal growth and cell division of Escherichia coli K-12. Molecular microbiology. 69, 870-81. 
Zhao, G., Winkler, M. E., 1996. A novel alpha-ketoglutarate reductase activity of the serA-encoded 3phosphoglycerate dehydrogenase of Escherichia coli K-12 and its possible implications for human 2hydroxyglutaric aciduria. Journal of bacteriology. 178, 232-9.

Zhu, Q., Zhang, X., Luo, Y., Guo, W., Xu, G., Shi, J., Xu, Z., 2015. L-Serine overproduction with minimization of by-product synthesis by engineered Corynebacterium glutamicum. Applied microbiology and biotechnology. 99, 1665-73.

Table 1: Mutations observed in strains isolated from three populations. Mutations in thrA highlighted in dark grey were reintroduced in parent strain using the $c a t-s a c B$ system. Mutations in light grey were reverse engineered by MAGE.

\begin{tabular}{|c|c|c|c|c|c|c|c|c|c|c|}
\hline Position & $\begin{array}{c}\text { Sequence } \\
\text { Change }\end{array}$ & Gene & Protein change & Q1 & $3-1$ & 3-2 & 4-1 & 4-2 & $5-1$ & $5-2$ \\
\hline 1403 & $\mathrm{~A} \rightarrow \mathrm{G}$ & thrA & $\mathrm{Y} 356 \mathrm{C}(\mathrm{TAC} \rightarrow \mathrm{TGC})$ & & & & & & & + \\
\hline 1405 & $\mathrm{~A} \rightarrow \mathrm{C}$ & thrA & S357R $(\mathrm{AGC} \rightarrow \mathrm{CGC})$ & & + & + & & & & \\
\hline 1413 & $\mathrm{~T} \rightarrow \mathrm{A}$ & thrA & S359R $($ AGT $\rightarrow$ AGA $)$ & & & & + & + & & \\
\hline 121518 & IS5 (+) & $\operatorname{aroP}$ & coding (31-34/1374 nt) & & + & + & & & & \\
\hline 450073 & $\mathrm{C} \rightarrow \mathrm{T}$ & $c y o B$ & $\mathrm{~W} 190 *(\mathrm{TGG} \rightarrow \mathrm{TAG})$ & + & & & + & + & + & + \\
\hline 822421 & $\mathrm{C} \rightarrow \mathrm{T}$ & $y b h N$ & L26L (TTG $\rightarrow$ TTA) & & & & & & + & + \\
\hline 932321 & $\mathrm{C} \rightarrow \mathrm{T}$ & $\operatorname{trx} B / l r p$ & intergenic (-271/-274) & & & & + & + & & \\
\hline 933022 & $\mathrm{~A} \rightarrow \mathrm{G}$ & $\operatorname{lrp}$ & $\mathrm{D} 143 \mathrm{G}(\mathrm{GAC} \rightarrow \mathrm{GGC})$ & & & & & & + & + \\
\hline 1756445 & $\mathrm{G} \rightarrow \mathrm{T}$ & $p y k F$ & $\mathrm{E} 250 *(\mathrm{GAA} \rightarrow \mathrm{TAA})$ & & + & + & & & & \\
\hline 1818103 & $\mathrm{~A} \rightarrow \mathrm{T}$ & $c h b F$ & V133E $($ GTA $\rightarrow$ GAA $)$ & + & + & + & + & + & + & + \\
\hline 2025007 & $\mathrm{~A} \rightarrow \mathrm{C}$ & yodD & $\mathrm{S} 8 \mathrm{R}(\mathrm{AGC} \rightarrow \mathrm{CGC})$ & & + & + & & & & \\
\hline 2307616 & $\mathrm{C} \rightarrow \mathrm{G}$ & yojI & $\mathrm{D} 334 \mathrm{H}(\mathrm{GAT} \rightarrow \mathrm{CAT})$ & & & & & + & & \\
\hline 2492323 & IS1 (-) & frc & coding (924-932/1251 nt) & & & & & & + & + \\
\hline 2599861 & $(\mathrm{~T}) 8 \rightarrow 7$ & $\operatorname{dapA} / g c v R$ & intergenic $(-101 /-45)$ & & + & + & + & + & & \\
\hline 2815907 & $\mathrm{G} \rightarrow \mathrm{A}$ & $g s h A$ & $\mathrm{~A} 178 \mathrm{~V}(\mathrm{GCG} \rightarrow \mathrm{GTG})$ & & & + & & & & \\
\hline 2907364 & $\mathrm{~A} \rightarrow \mathrm{T}$ & eno & L193Q $(\mathrm{CTG} \rightarrow \mathrm{CAG})$ & + & + & + & + & + & + & + \\
\hline 2907452 & $\mathrm{C} \rightarrow \mathrm{G}$ & eno & V164L $(\mathrm{GTT} \rightarrow \mathrm{CTT})$ & & & & & & + & + \\
\hline 2942635 & $(\mathrm{~T}) 7 \rightarrow 6$ & $g c v A / g c v B$ & intergenic (-68/-61) & & & & + & + & & \\
\hline 2942878 & IS4 (+) & $g c v A / g c v B$ & coding (180-182/208 nt) & & + & + & & & & \\
\hline 3060146 & $\mathrm{C} \rightarrow \mathrm{A}$ & $\arg P$ & $\mathrm{Q} 132 \mathrm{~K}(\mathrm{CAG} \rightarrow \mathrm{AAG})$ & & & & & & + & + \\
\hline 3471274 & $\mathrm{C} \rightarrow \mathrm{A}$ & tufA & G19V $(\mathrm{GGC} \rightarrow \mathrm{GTC})$ & & & & & & + & + \\
\hline 3514455 & $\mathrm{~A} \rightarrow \mathrm{G}$ & rpe & $\mathrm{I} 202 \mathrm{~T}(\mathrm{ATC} \rightarrow \mathrm{ACC})$ & & & & + & + & & \\
\hline 3554342 & $\mathrm{C} \rightarrow \mathrm{T}$ & malT & $\mathrm{Q} 420 *(\mathrm{CAA} \rightarrow \mathrm{TAA})$ & & + & & & & & \\
\hline 3966174 & IS1 (-) & $\operatorname{trx} A /$ rho & intergenic $(+84 /-235)$ & & & & & & + & + \\
\hline 3966676 & $\mathrm{G} \rightarrow \mathrm{T}$ & rho & $\mathrm{R} 87 \mathrm{~L}(\mathrm{CGC} \rightarrow \mathrm{CTC})$ & & & & + & + & & \\
\hline 4182803 & $\mathrm{C} \rightarrow \mathrm{T}$ & rров & P520L (CCG $\rightarrow$ CTG $)$ & & + & + & & & & \\
\hline 4248304 & $\mathrm{C} \rightarrow \mathrm{T}$ & $\operatorname{lam} B$ & $\mathrm{Q} 112 *(\mathrm{CAG} \rightarrow \mathrm{TAG})$ & & & + & & & & \\
\hline 4428871 & $\mathrm{~T} \rightarrow \mathrm{C}$ & $y t f B / f k l B$ & intergenic (-154/-64) & & & & + & + & & \\
\hline
\end{tabular}




\section{Highlights}

- Adaptive laboratory evolution enhanced tolerance towards L-serine from 3 to $100 \mathrm{~g} / \mathrm{L}$

- Potential site of L-serine inhibition identified on homoserine dehydrogenase, ThrA

- Gene clusters encoding potential serine exporters duplicated in evolved strains

- Combined MAGE and amplicon sequencing enables identification of causative mutations

- Evolved strain produced $37 \mathrm{~g} / \mathrm{L}$ of L-serine with a $24 \%$ mass yield from glucose

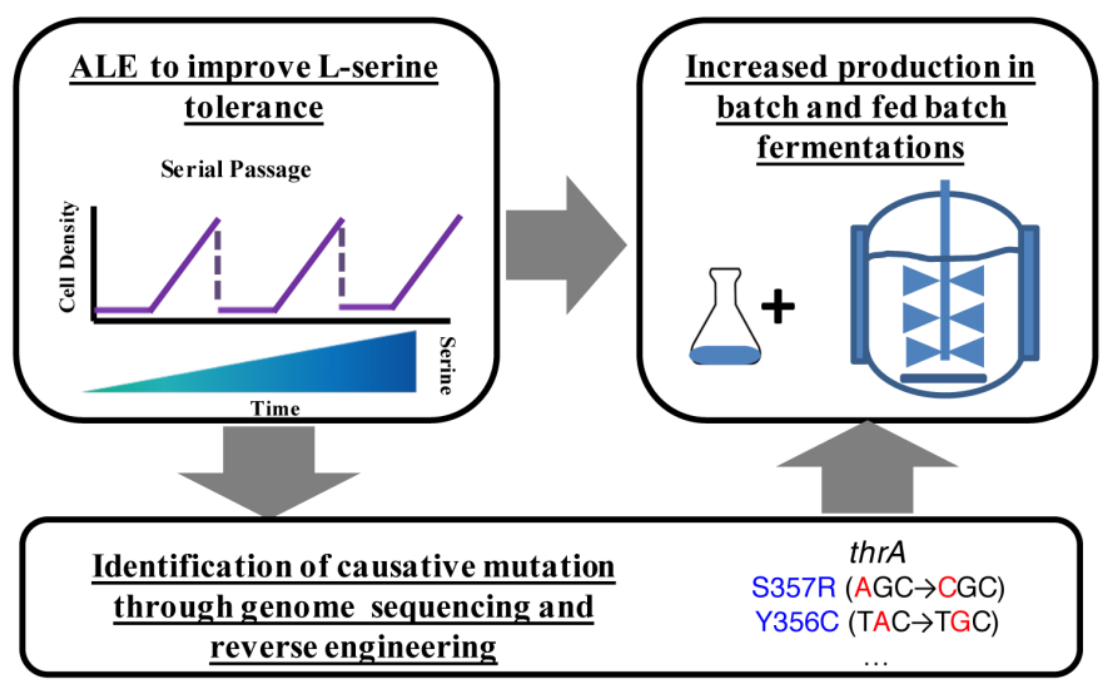

